\title{
Comparison of touch- and laser heat-evoked cortical field potentials in conscious rats
}

\author{
Fu-Zen Shaw ${ }^{\text {a }}$, Ruei-Feng Chen ${ }^{\mathrm{b}}$, Hen-Wai Tsao ${ }^{\mathrm{a}}$, Chen-Tung Yen ${ }^{\mathrm{b}, *}$ \\ a Department of Electrical Engineering, National Taiwan University, Taipei, Taiwan \\ ${ }^{\mathrm{b}}$ Department of Zoology, National Taiwan University, No. 1, Section 4, Roosevelt Road, Taipei 106, Taiwan
}

Accepted 26 January 1999

\begin{abstract}
Field potentials and multiunit activities from chronically implanted cortical electrodes were used to study tactile and nociceptive information processing from the tail of the rat. Fourteen stainless steel screws implanted in the skull were used as electrodes to record field potentials in different cortical areas. Electrical, mechanical, and laser pulses were applied to the tail to induce evoked cortical field potentials. Evoked responses were compared before and after sodium pentobarbital anesthesia (50 mg/kg, i.p.). In both electrical- and mechanical-evoked potential (EEP and MEP) studies, two major peaks were found in the conscious animal. The polarity of the late component was modified after pentobarbital anesthesia. In the laser-evoked potential (LEP) study, two distinct negative peaks were found. Both peaks were very sensitive to anesthesia. Following quantitative analysis, our data suggest that the first positive peak of EEP and MEP corresponded to the activation of the A $\beta$ fiber, the second negative peak of MEP and the first peak of LEP corresponded to A $\delta$ fiber activation, while the second peak of LEP corresponded to C fiber activation. The absolute magnitudes of all cortical components were positively related to the intensity of the stimulation. From spatial mapping analysis, a localized concentric source of field potential was observed in the primary somatosensory cortex (SI) only after activation of the A $\beta$ fiber. Larger responsive cortical areas were found in response to $\mathrm{A} \delta$ and $\mathrm{C}$ fiber activation. In an intracortical recording experiment, both tactile and nociceptive stimulation evoked heightened unit activity changes at latencies corresponding to respective field potentials. We conclude that different cortical areas are involved in the processing of A and C fiber afferent inputs, and barbiturate anesthesia modifies their processing. (C) 1999 Elsevier Science B.V. All rights reserved.
\end{abstract}

Keywords: A fiber; C fiber; Topographical mapping; Nociception; Somatosensory function; Electrical stimulation

\section{Introduction}

Early in this century, Head and Holms [17] observed that the threshold for pain stimuli is preserved after damage to the cerebral cortex, but that pain thresholds and pain sensation change with damage to the thalamus in patients. These observations have led to the still widely held view that pain is sensed in the thalamus. Over the past two decades, however, results from humans [4,18,20,24,25,37], primates $[16,22]$, cats $[30,32]$, and rats $[18,21,28,29,39]$ have indicated that the cerebral cortex participates in pain processing. Electrophysiological evidence from painful

\footnotetext{
"Corresponding author. Fax: +886-2-2363-6837; E-mail: ctyen@ccms.ntu.edu.tw
}

electrical stimulation (transcutaneous stimulation, toothpulp stimulation) or laser heat stimulation has been reported in the neocortex of anesthetized monkeys [23,24], cats [30,32], and rats $[18,21,28,29,33]$. It is well known, however, that anesthesia strongly modifies nociceptive responses $[8,13,26,38]$. One of the goals of the present study is to evaluate nociceptive-evoked responses in awake rats.

Short-pulse noxious $\mathrm{CO}_{2}$-laser radiation activates mainly $\mathrm{A} \delta$ and $\mathrm{C}$ nociceptors in rats [15] and humans [3,5], without activating low-threshold mechanoreceptors. In humans, $\mathrm{CO}_{2}$-laser stimulation-evoked potentials (LEPs) have been recorded from the scalp, and such potentials are assumed to be of cerebral origin. Latencies of these potentials correspond to activation of $\mathrm{A} \delta$ and $\mathrm{C}$ fibers $[4,7,9]$. These evoked potentials coincide with the perception of pricking and burning pains, respectively [4,7]. Similar 
evoked potentials using $\mathrm{CO}_{2}$-laser radiation have been recorded in rats under halothane anesthesia [21]. In that study, Kalliomaki et al. [21] reported that late cortical LEP evoked from laser stimulation of glabrous skin of paws of rats corresponded to $\mathrm{C}$ fiber velocity. This late LEP occupies a larger area of the sensorimotor cortex as compared to the short latency-evoked potential elicited from innocuous mechanical stimulation. Using standard electrophysiological recording techniques, they found that the late LEP exhibited two intracortical foci: one at laminae III/IV and the other at laminae V-VI.

The major purpose of the present study was to use laser heat irradiation to study the processing of nociceptive input in the cerebral cortex and to compare LEPs with low-intensity tactile- and electrical stimulation-evoked potentials (EEPs) in conscious, awake rats. Topographical distributions of these LEPs were determined with $14 \mathrm{im}$ planted stainless steel screws evenly spaced over the top of the skull. To ascertain that these evoked potentials were of cortical origin, intracortical recording was performed with multichannel microwire bundles whose tips were exposed at different depths of the cortical layers. Preliminary results have been presented in abstract form [34].

\section{Materials and methods}

\subsection{Implantation of surface electrodes}

Adult male Wistar rats $(250-300 \mathrm{~g})$ were anesthetized initially with sodium pentobarbital $(50 \mathrm{mg} / \mathrm{kg}$ b.wt.). Ketamine was administrated as necessary to maintain proper anesthetic depth during surgery. The dorsal surface of the animal's head was shaved and placed in a stereotaxic apparatus. A midline scalp incision was made. Connective tissue was scraped away from the skull surface. The skull was roughened with a bone curette and then cleaned with saline. After exposure of the skull, a small concavity on each marked position of the skull was drilled first and then a stainless steel screw (O.D. $=1 \mathrm{~mm}$ ) was securely driven into this position. The small screw electrodes were positioned and symmetrically arranged $3 \mathrm{~mm}$ apart on both sides of the skull. The location of the recording channels is shown in the inset of Fig. 6. These skull electrodes did not penetrate the underlying dura. The impedance of these electrodes was $1.5-10 \mathrm{k} \Omega$. Monopolar recordings were made from screws relative to the reference electrode, which was implanted $2 \mathrm{~mm}$ caudal to the lambda at the midline. Individual screws were connected to a pin in a connector module. To assure functioning of electrodes after surgery, evoked potentials were elicited by stimulating the tail of the rat using a 0.5 -ms duration constant electrical current pulse (Grass S44). Subjects without identifiable evoked potentials or with evoked potentials of negative polarity in channel 5 or 9 , which overlay the tail representative area of the primary somatosensory cortex (SI) (Fig. 6, Ref. [14]) and indicate penetration into the cerebral cortex, were eliminated from the study. Dental cement was then applied and the connection module was fastened to the surface of the skull. Finally, animals were injected with antibiotics (Combion-S, 60,000 U/kg, i.p.) and housed individually in cages.

\subsection{Implantation of microwire bundle}

Formvar-insulated nichrome microwire (A-M systems; \#7610, $18 \mu \mathrm{m}$ diam.) was used to record the activity of SI neurons. In order to record the activity at different cortical layers, the microwire bundle was carefully arranged. A glass micropipette with a long shaft $(5 \mu \mathrm{m}$ diam.) was made with a horizontal pipette puller (Sutter Instrument model P-87). With this micropipette serving as a centerpiece, eight microwires were glued spirally in sequence onto the micropipette at different depths under a stereomicroscope. The total width of the 8-channel microwire bundle was about $100 \mu \mathrm{m}$. Each microwire was soldered individually to a connection module. Prior to implantation, the cortical representative area of the tail was mapped using a glass microelectrode filled with a $3-\mathrm{M} \mathrm{NaCl}$ solution. The microwire bundle was then implanted at the location with maximal cortical response to tail stimulation.

Two weeks following recovery from surgery, individual animals were placed in an acrylic recording chamber with a slit in the top. This chamber was designed to allow recording from unrestrained animals. The behavior of the rat could be clearly observed. We used a modified multistrand printer cable line to connect the connection modules to the skull and amplifiers through the slit in the top cover of the chamber. To habituate rats to the experimental chamber, each animal was placed in a recording chamber 5 times $(2 \mathrm{~h} /$ day $)$ before the experiment. On the day of the actual recording, a 30-min period was allowed for the rat to become familiar with the environment. Surgical and recording procedures complied with NIH (USA) recommended procedures for animal use and care.

\subsection{Tail stimulation}

Three types of somatic stimuli were used: electrical stimulation, mechanical stimulation, and $\mathrm{CO}_{2}$-laser irradiation. For electrical stimulation, multistrand stainless steel wires were wrapped around the tail base. They were held in place with adhesive tape, and electrode cream was applied to enhance the contact. Constant electrical current pulses of 2-ms duration (Grass S44) were delivered to the tail. The intensity of the electrical current was set in the range from 0.1 to $0.5 \mathrm{~mA}$. This current range was chosen for the activation of low threshold cutaneous receptors. For mechanical stimulation, a blunt probe $(5 \mathrm{~mm}$ diam.) attached to a coil was used. The probe was displaced by a 
short-lasting current pulse (2 ms) generated by a Grass stimulator. The stimulator was adjusted to cause light pressure of the skin $(15-20 \mathrm{~g})$. The interstimulus interval of electrical and mechanical stimulation was kept at $1 \mathrm{~s}$. To lessen the contamination of the recording, the stimulus was interrupted when the rat was active (such motion as grooming, chewing, etc.).

The skin was stimulated with radiant heat pulses emitted from a $\mathrm{CO}_{2}$-laser (medical surgical laser, Tjing Ling \#2, National Taiwan University, $10.6 \mu \mathrm{m}$ wavelength) operating in $\mathrm{TEM}_{00}$ mode (Gaussian distribution) [39]. The beam diameter was $3 \mathrm{~mm}$ (unfocused). The duration of the stimulation pulse was kept at $10 \mathrm{~ms}$. The output power was between 6 and $18 \mathrm{~W}$ [39]. These output energies correspond to $60-180 \mathrm{~mJ}$. These stimulation energies did not result in any visible damage to the skin. To minimize tissue damage, sensitization and habituation, the stimuli were randomly applied to a local skin area $(1 \mathrm{~cm}$ for $\mathrm{Tb}$ and $\mathrm{Tm} ; 1.5 \mathrm{~cm}$ for $\mathrm{Tp}$ ). The interstimulus interval was longer than $5 \mathrm{~s}$. With each laser stimulation, a jerky movement of the tail was noted. For tail-tip and midtail stimulation, this movement was a flick of the tail; for tail-base stimulation, the observed movement was an arching or curling of the tail. Both response types were used in the calculation of the proportion of tail flick. Effects of pentobarbital on these somatic-evoked potentials were tested in a few rats (see Section 3). In these animals, sodium pentobarbital $(50 \mathrm{mg} / \mathrm{kg})$ was administered i.p.; the same somatic stimuli were applied before and after pentobarbital anesthesia.

\subsection{Recording and data analysis}

Field potentials from skull electrodes were amplified with a 16-channel amplifier (4th order bandpass filter: $0.3-1000 \mathrm{~Hz}$, gain $=5000 \times)$. These multichannel potentials were digitized with a data acquisition card (AT-16MIO-F5, National Instruments, Austin, TX) with the external trigger locked. Sampling frequency for individual channels was $4 \mathrm{kHz}$. Unit activity was amplified in the frequency range of $300-3000 \mathrm{~Hz}(20,000 \times)$. The sampling frequency was $20 \mathrm{kHz}$ for individual channels. Data were stored on a computer hard disk and trials were averaged on-line. Each evoked potential was selected from an average of about 100 stimuli. Evoked data trials were rejected from averaging if they showed a contaminated movement artifact, large baseline shift, or spindle rhythm. To evaluate the spatial pattern of the responses, a topographical map was used. Topographical maps were calculated with weight average interpolation [31,35]. Details of the analytical program were described in a previous investigation [35]. Intracortical multiunit recordings were either rectified and integrated (integrated multiunit activity, IMUA), or passed through a window discriminator. Numbers of spikes and frequencies from the window discriminator were calculated and analyzed with a software pro- gram developed on a LabVIEW (National Instruments, Austin, TX) environment.

\subsection{Histological procedures}

After completion of the stimulation and recording experiment, the animal was overdosed with sodium pentobarbital and subsequently perfused with $10 \%$ formalin in saline. Special attention was given to ascertain that stainless screws did not penetrate the dura. Serial $50-\mu \mathrm{m}$ frozen sections of the brain were cut and stained with thionin. The location of the microwire bundle was determined with microscopic examination of the stained sections and documented with a camera lucida attachment.

\section{Results}

\subsection{Cortical responses to different somatic stimulation}

Three different types of somatic stimulation were used on tails of rats. Three different sites on the tail (tail base [Tb], middle tail $[\mathrm{Tm}]$, and tail tip [Tp]) were given mechanical and $\mathrm{CO}_{2}$-laser stimulation. The electrical stimulation was applied only to the tail base of the rat. Examples of field potentials elicited in the tail representative area of SI, evoked by mechanical and $\mathrm{CO}_{2}$-laser stimulation at these 3 sites, are shown in Fig. 1. For mechanical stimulation, two major components (Fig. 1, left column) were observed: a biphasic waveform (positive peak followed by a negative peak) followed by a larger negative peak. For $\mathrm{CO}_{2}$-laser radiation, two components were also observed: a biphasic waveform followed by a negative peak (Fig. 1, right column). A cortical-evoked potential of biphasic waveform (positive peak followed by a negative peak) followed by a larger negative peak was observed following electrical stimulation (indicated by arrowheads in Fig. 2A).

Under pentobarbital anesthesia, the first component of mechanical-evoked potential (MEP) was enhanced ( $n=8$, $p<0.001$ ) while the second components of MEP was reversed in waveform (dotted line in middle panel of left column of Fig. 1). In contrast, all peaks of LEP were abolished during the first 90 min after pentobarbital anesthesia. Two hours after pentobarbital administration, the rat showed spontaneous twitching movements and displayed other signs of returning to consciousness. However, cortical responses did not completely recover. Interestingly, with the same laser irradiation, the late component of LEP changed to a positive peak (dotted line in middle panel of right column of Fig. 1). Similar to the MEP changes, the first component of the EEP was enhanced, while the late component was changed $2 \mathrm{~h}$ after pentobarbital injection (dotted line in Fig. 2A). 


\section{Mechanical}

$\mathrm{Tp}$

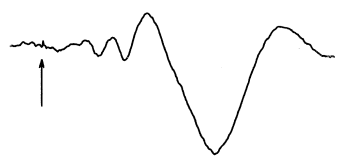

$\mathrm{Tm}$

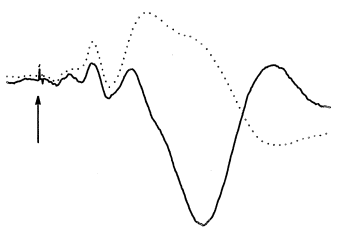

$\mathrm{Tb}$

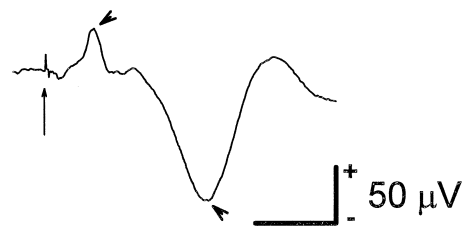

$25 \mathrm{~ms}$
$\mathrm{CO}_{2}$-Laser
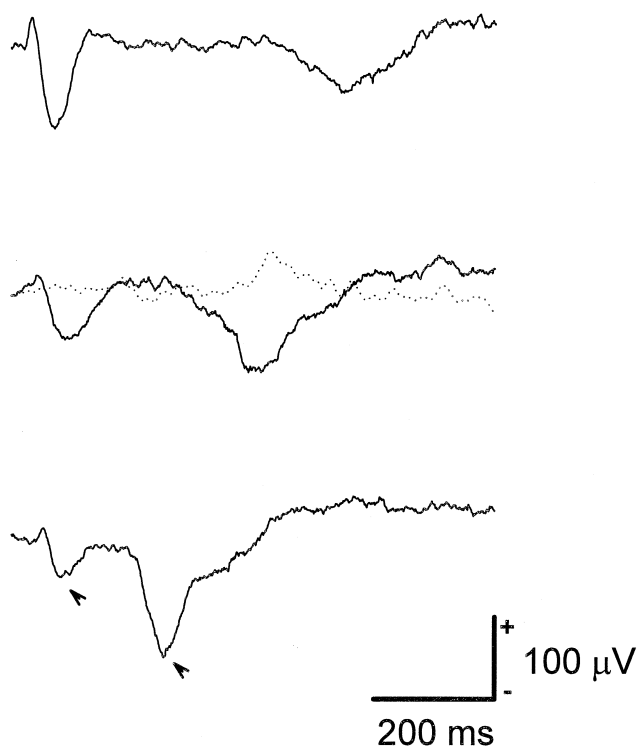

Fig. 1. Examples of MEPs (left column) and LEPs (right column) of SI evoked from stimulation at three different tail sites in awake (solid line) and anesthetized (dotted line) conditions. Intensities of $\mathrm{CO}_{2}$-laser stimulation at the base of the tail ( $\mathrm{Tb}$ ), middle tail $(\mathrm{Tm})$, and tip of tail ( $\mathrm{Tp}$ ) were 12,12 , and $15 \mathrm{~W}$, respectively. The major components of MEP and LEP are indicated by arrowheads in the bottom traces. Two hours after sodium pentobarbital injection $(50 \mathrm{mg} / \mathrm{kg}$, i.p.), patterns of MEP and LEP following Tm stimulation were still modified. The long arrows in the left column indicate the tactile stimulus artifact.

\subsection{Peripheral afferent fibers mediating different evoked potentials}

To estimate the conduction velocity of the peripheral afferent fibers mediating the MEPs and LEPs in freely moving rats, the latency of these potentials to three different sites of the tail was measured. These latencies were used to estimate the peripheral conduction velocity of the major peaks in MEP and LEP. The average latencies (mean \pm S.E.) of the first positive peak evoked from

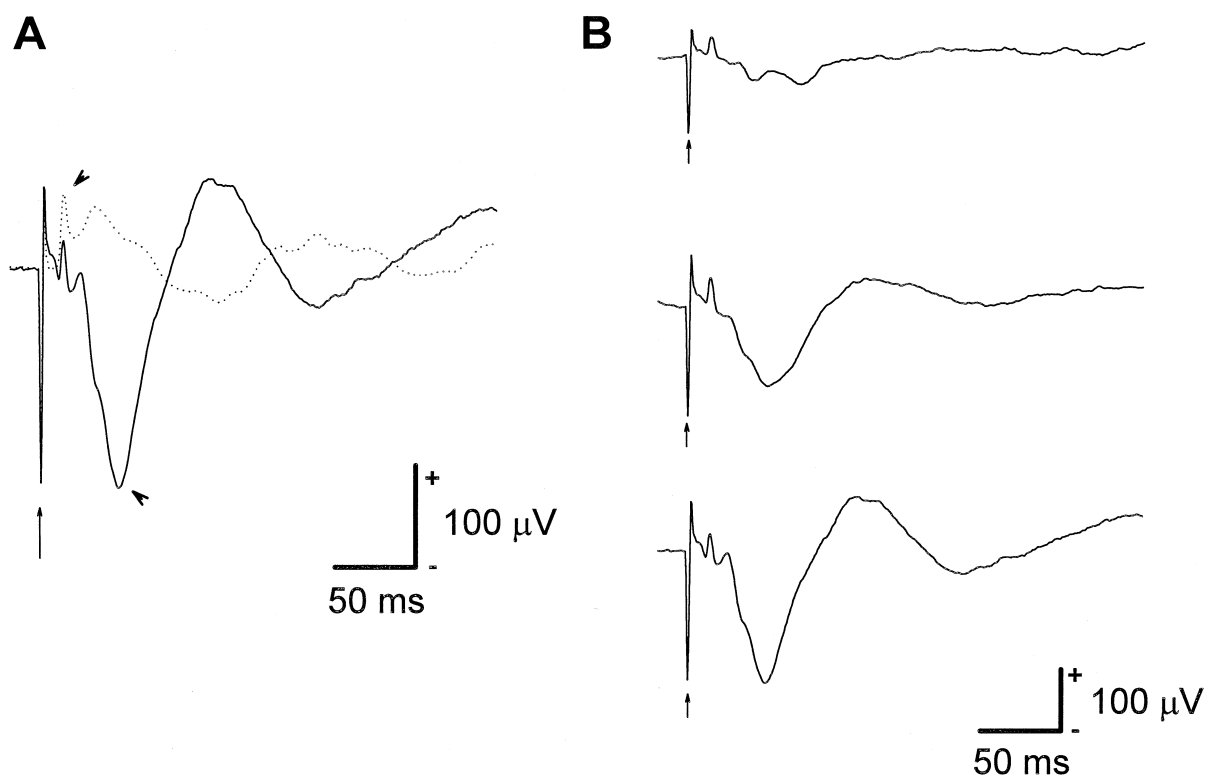

Fig. 2. Examples of EEPs after stimulation at tail base. (A) EEPs in awake (solid line) and anesthetized (dotted line) conditions. The intensity of the electrical current was $0.5 \mathrm{~mA}$ with a 2-ms duration. The major components are indicated by arrowheads. (B) EEPs following stimulation at three different intensities. The stimulation current intensities $(0.2,0.3$, and $0.5 \mathrm{~mA})$ increase from top to bottom traces. Small arrows indicate the stimulus artifact. 
$\mathrm{Tb}, \mathrm{Tm}$, and $\mathrm{Tp}$ following mechanical stimulation were $15.6 \pm 0.6,16.4 \pm 0.8$, and $21.4 \pm 0.8 \mathrm{~ms}$, respectively. We found that peak latency increased linearly with conduction distance along the tail (Fig. 3). Estimation of conduction velocity with the slope of the linear regression line was $35 \mathrm{~m} / \mathrm{s}(p<0.001)$. For the second component of the MEP, average latencies evoked from $\mathrm{Tb}$, Tm, and Tp were $49.5 \pm 2.0,50.8 \pm 1.0$, and $56.5 \pm 1.7 \mathrm{~ms}$, respectively. Estimation of conduction velocity was $28 \mathrm{~m} / \mathrm{s}$ $(p<0.005)$. Latencies of the first negative peak evoked from $\mathrm{Tb}, \mathrm{Tm}$, and $\mathrm{Tp}$ following laser stimulation were $70.6 \pm 2.9,73.8 \pm 2.6$, and $79.6 \pm 3.5 \mathrm{~ms}$, respectively. Conduction velocity was approximately $22 \mathrm{~m} / \mathrm{s}(p<$ $0.05)$. Latencies of the second negative peak from $\mathrm{Tb}, \mathrm{Tm}$, and $\mathrm{Tp}$ following laser stimulation were $246.7 \pm 8.0,394.1$ \pm 14.5 , and $519.5 \pm 22.7 \mathrm{~ms}$, respectively. Conduction velocity was $0.7 \mathrm{~m} / \mathrm{s}(p<0.001)$. Conduction velocity of the first positive peak to mechanical stimulation was mediated by the $A \beta$ fiber. Conduction velocities of the second negative peak of MEP and the first negative peak of LEP were in the range of the A $\delta$ fiber. The second negative peak of LEP was mediated by the $\mathrm{C}$ fiber.

\subsection{Stimulus-response relations of EEP and LEP}

Four different current intensities $(0.1,0.2,0.3$, and 0.5 $\mathrm{mA}$ ) were used to evaluate the stimulus-response relationships in free-moving rats $(n=8)$. Fig. $2 \mathrm{~B}$ shows EEP
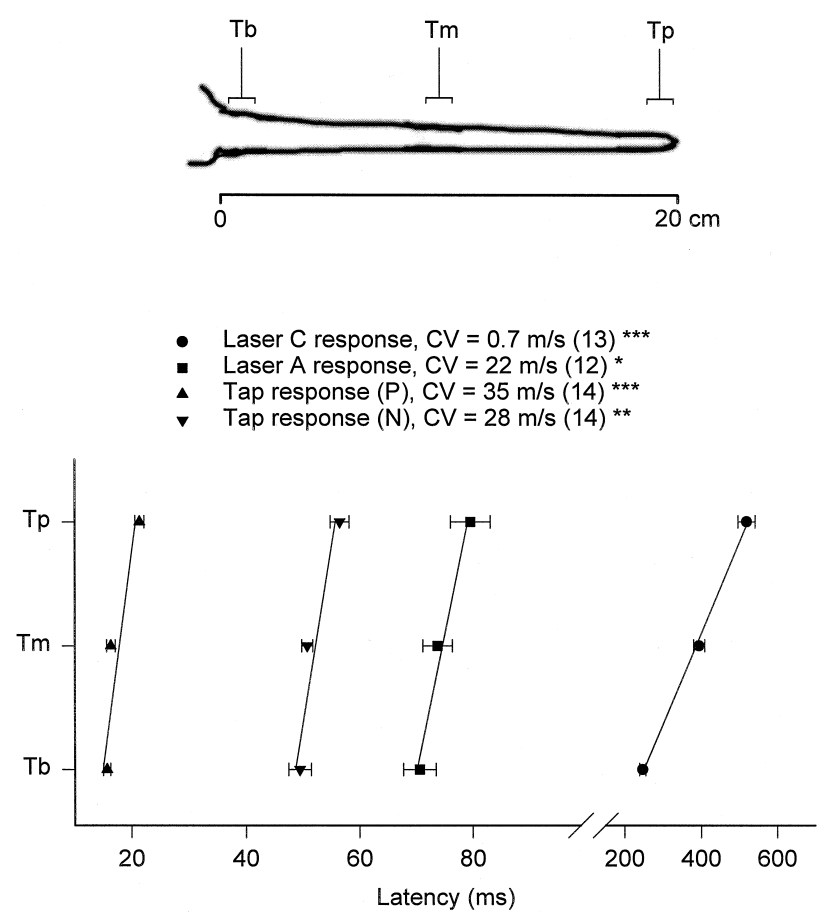

Fig. 3. Relationship between conduction distance and peak latencies of MEPs and LEPs. The slopes of the linear regression line of the four components are 35, 28, 22, and $0.7 \mathrm{~m} / \mathrm{s}$, respectively. Data are presented as mean \pm S.E. $* p<0.05 ; * * p<0.01 ; * * * p<0.001$ (Student's $t$ test).
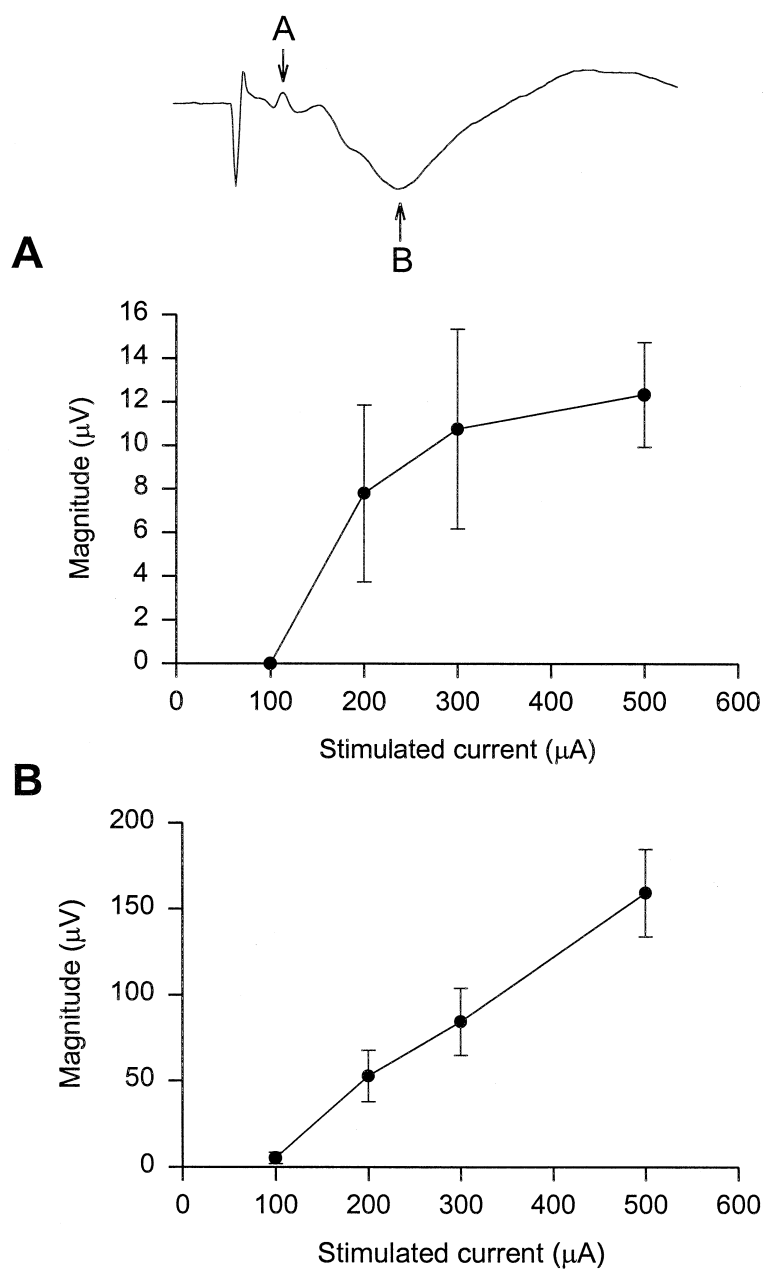

Fig. 4. Changes in magnitude (mean \pm S.E.) of EEPs evoked from different current intensity stimulation at the tail base. (A) Magnitude of the positive component of EEP increases as stimulus current increases. A sigmoidal relationship was found between current intensity and EEP magnitude. (B) The magnitude of the negative component of EEP increases linearly with increases in stimulus current.

traces under $0.2,0.3$, and $0.5 \mathrm{~mA}$ current stimulation. The magnitude (mean \pm S.E.) of the first and second component of the EEP at each stimulus intensity is shown in Fig. 4. Amplitudes of both positive (Fig. 4A) and negative peaks (Fig. 4B) of EEPs became larger as the stimulation current increased. The threshold current for EEP was between 0.1 and $0.2 \mathrm{~mA}$. The amplitude of the positive component of EEP showed a sigmoidal curve with increasing current intensity. The amplitude of the negative component of EEP showed a linear relation to the stimulated current. We did not apply intensities higher than $0.5 \mathrm{~mA}$, because at this intensity rats sometimes indicated uncomfortable behavior (vocalization and/or biting the bandage).

Five different laser energies (60, 90, 120, 150, and 180 $\mathrm{mJ})$ were applied to tails of rats. The magnitude of LEPs $(n=9)$ and the proportion of tail flick $(n=11)$ vs. five different stimulus energies applied to the tail base, the middle tail, or the tail tip are shown in Fig. 5. The proportion of tail flick increased as the stimulus energy 

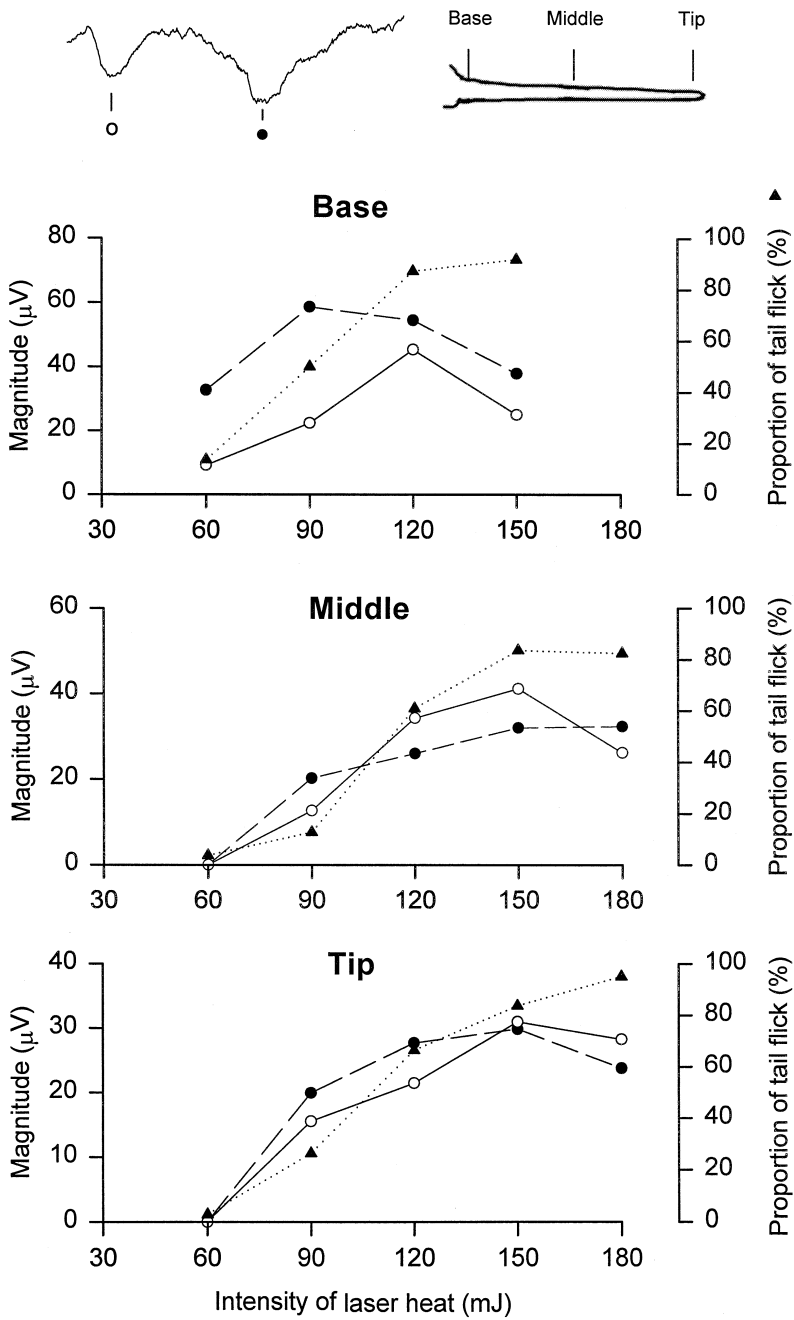

Fig. 5. Changes in magnitude of LEPs and the proportion of tail flick after laser heat indication for different tail regions. The $x$-axis represents radiation heat energy. Note both the magnitude of LEPs (first negative component: open circles; second negative component: filled circles) and the proportion of tail flick (filled triangles) become larger as the stimulation energy increases.

increased (filled triangles). The magnitudes of both the first negative component (open circles) and the second negative component (filled circles) of LEPs increased as stimulus energy increased. The threshold energy of LEPs evoked from the tail base was lower than $60 \mathrm{~mJ}$. Threshold energies of LEPs evoked from the middle tail and tail tip were between 60 and $90 \mathrm{~mJ}$. The response amplitude of LEPs and the proportion of tail flick reached a maximum at an energy of about $120 \mathrm{~mJ}$ following tail base stimula- tion. The maximum response stimulation intensities for middle tail and tail tip stimulation were about $150 \mathrm{~mJ}$.

\subsection{Spatiotemporal distribution of stimulus-evoked poten- tials}

The topographical distribution of cortical potentials evoked by mechanical, electrical, and $\mathrm{CO}_{2}$-laser stimulation of the tail was studied. Examples of stimulation-evoked responses to the three types of stimulation are shown in Figs. 6-8. Evoked potentials recorded from the 12 recording sites to mechanical stimulation of the tail base in one rat are illustrated in Fig. 6A. The first biphasic waveform can be clearly seen in channels 5 and 9 (tail representative area of SI). The concentric source to first positive peak (latency $=15 \mathrm{~ms}$ ) was localized bilaterally over the tail representative area of SI (Fig. 6B). Bilaterally localized current sink of SI was present at the beginning stage of the large late negative component (latency $=30 \mathrm{~ms}$ ). The spatial distribution of the larger negative peak (latency $=50$ $\mathrm{ms}$ ) is shown at the lower right corner in Fig. 6B. This negative peak showed a localized contour central to the primary somatosensory area similar to the short positive peak. A similar phenomenon was also found in low intensity EEPs elicited by electrical stimulation. However, the topographical distribution of the slower negative EEP was much larger with stronger stimulation intensity $(0.5 \mathrm{~mA})$. At this intensity, the first biphasic waveform was localized in channels 5 and 9. The longer latency negative peak was, however, widely spread over several recording sites (Fig. 7).

Two distinct components of LEP following $\mathrm{CO}_{2}$-laser stimulation were found at all recording sites (Fig. 8A). The faster component was a biphasic waveform. The positive peak of the faster component $(48.5 \mathrm{~ms})$ had a bilateral concentric current sink at SI, followed by a widespread distribution of the negative peak in the frontal-parietal cortical area (Fig. 8B). The slower component $(245 \mathrm{~ms})$ also showed a widespread distribution in the topographical map (Fig. 8B).

Spatiotemporal characteristics of MEP, EEP, and LEP, including their polarity, latency, conduction velocity, and spatial distribution over the cortical surface, are summarized in Table 1.

\subsection{Cortical neuronal activities evoked by mechanical and laser stimulation}

To better understand the far-field or the near-field origin of MEP and LEP, cortical neuronal activities were

Fig. 6. Spatial distribution of MEPs. (A) An example of 12-channel recording of MEPs displayed in related recording sites over the skull (indicated at the inset). Each trace represents the average of 200 trials. The units of the $x$-axis and $y$-axis are $25 \mathrm{~ms}$ and $50 \mu \mathrm{V}$, respectively. (B) Topographical maps of MEPs. The upper trace is the MEP at channel 5 (see numbers in the inset of [A]). Four isopotential maps were made at latencies of 15, 20.5, 30, and 50 ms from the start of the mechanical stimulation, respectively. Note the localized potential peaks at the tail representation area of SI. The unit of the gray scale is in microvolts $(\mu \mathrm{V})$. The units of the horizontal and vertical scales are in millimeters $(\mathrm{mm})$. The zero point $(0,0)$ is at the bregma. REF: reference electrode; GND: ground. 

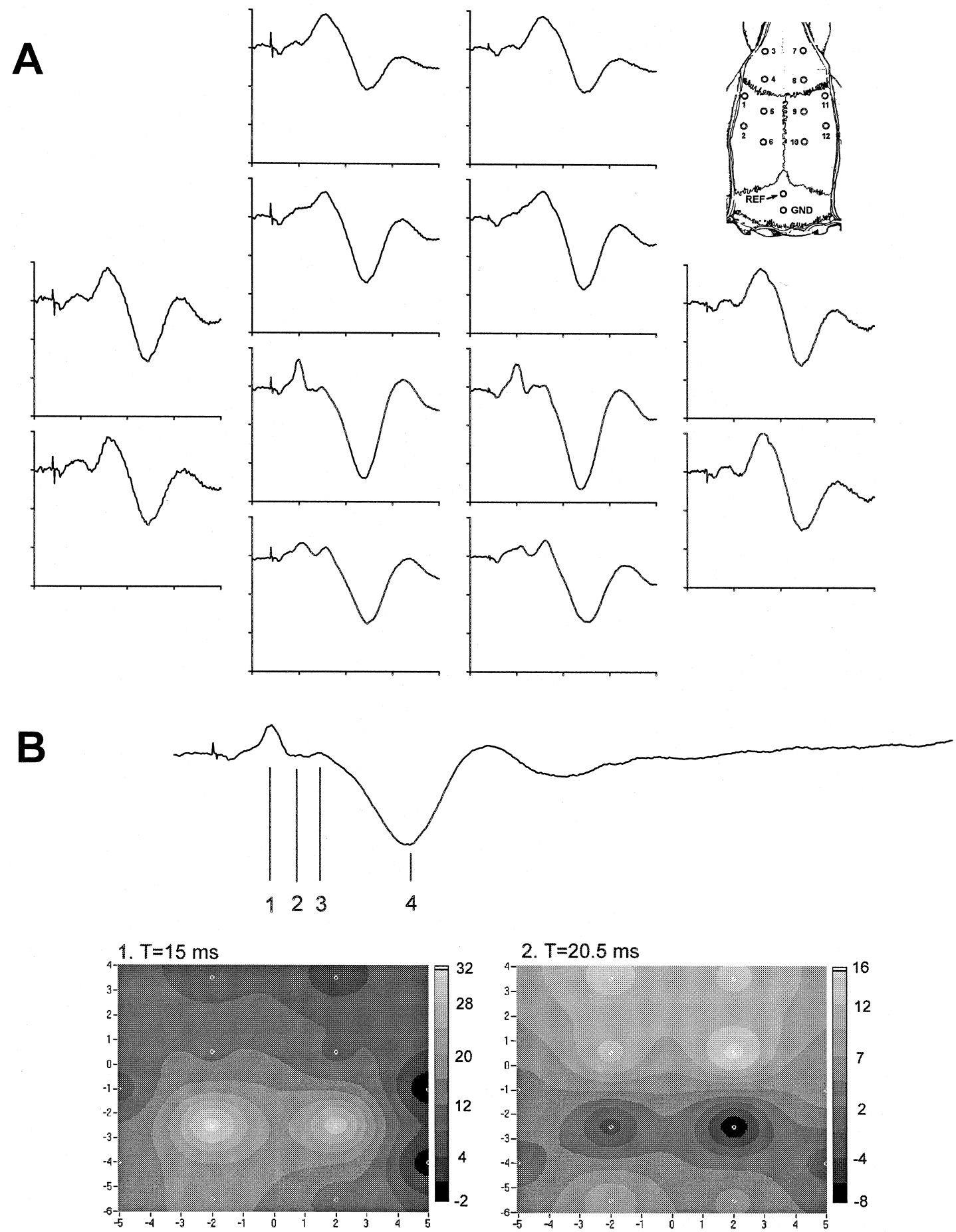

2. $T=20.5 \mathrm{~ms}$
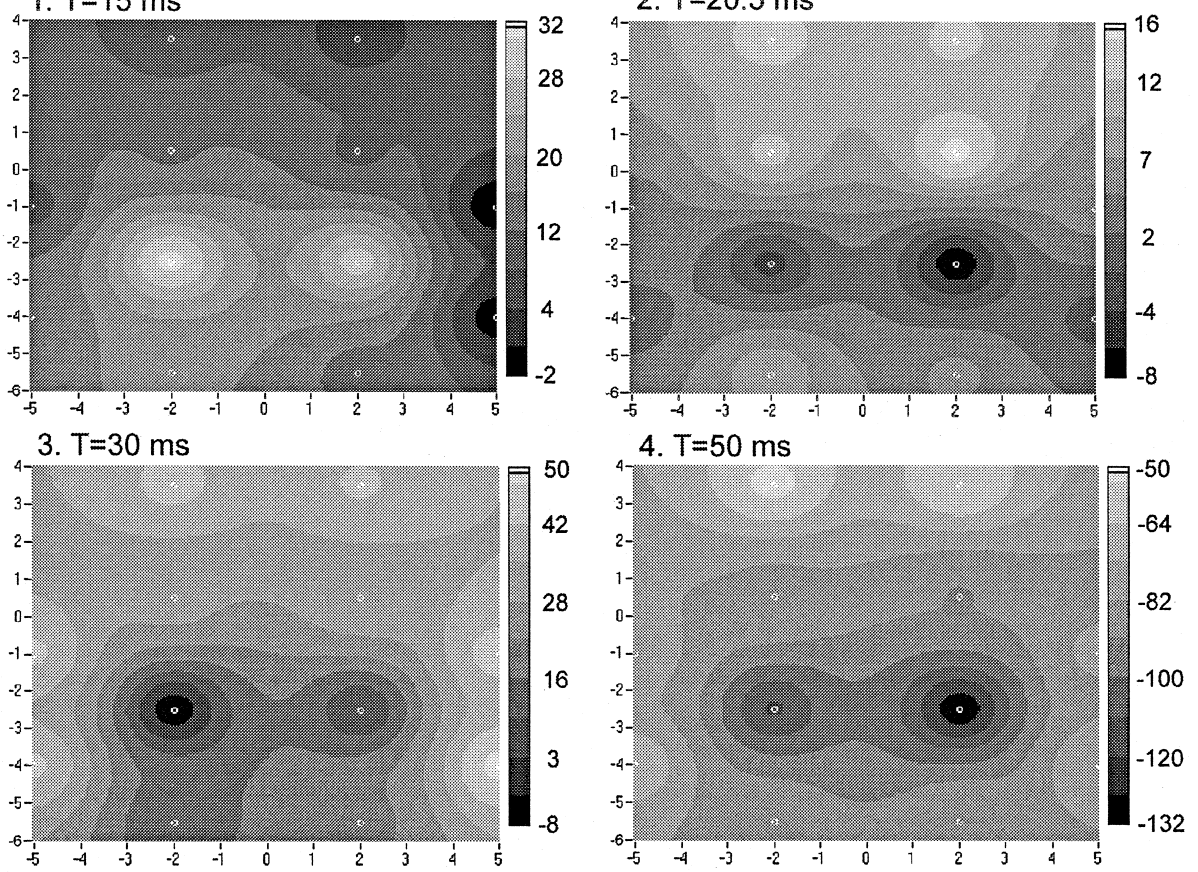


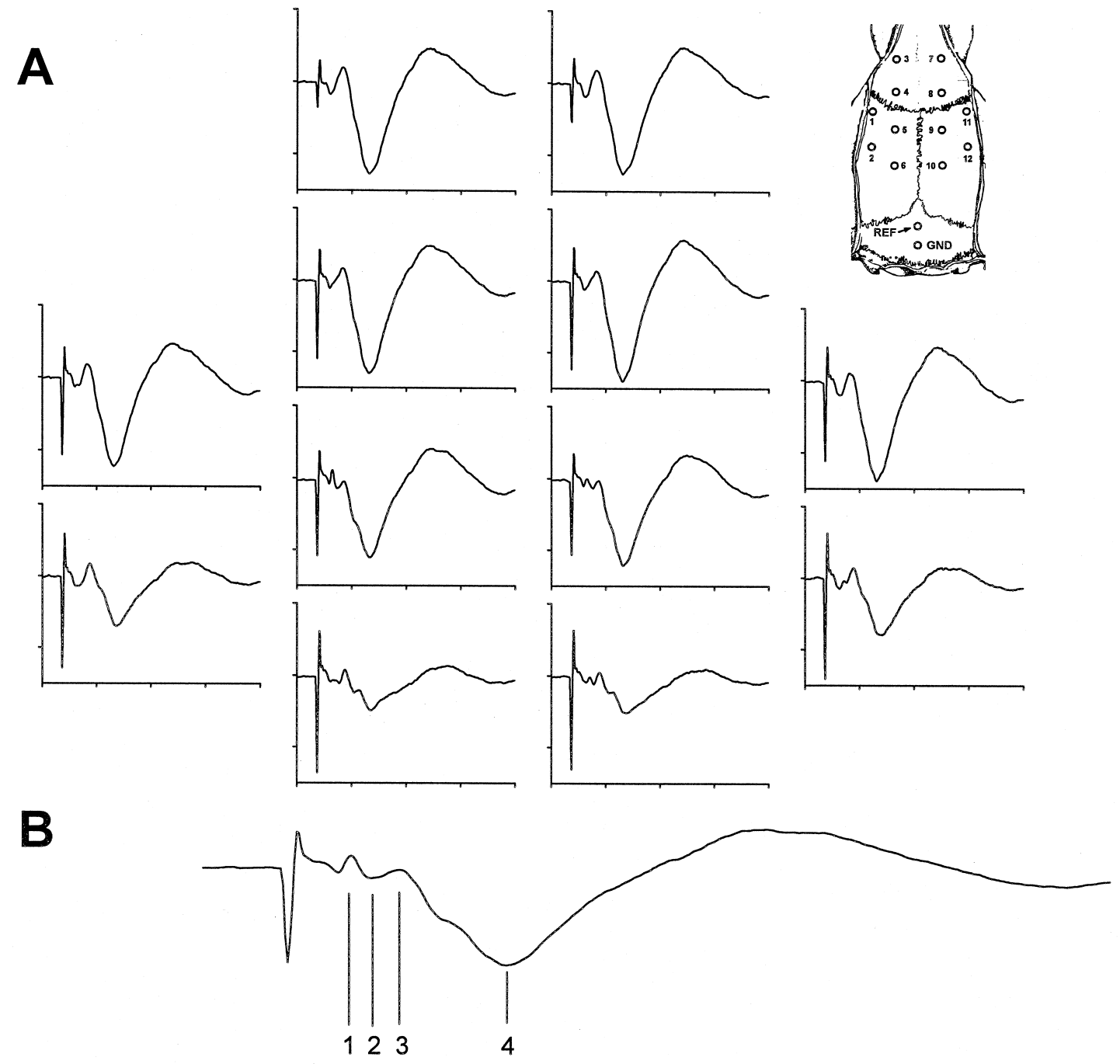

1. $T=16 \mathrm{~ms}$

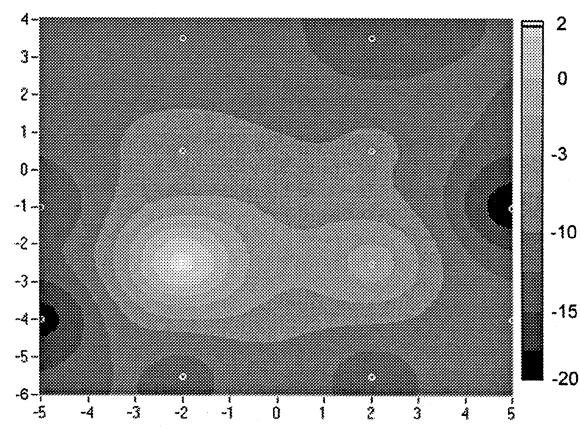

3. $\mathrm{T}=\mathbf{2 7} \mathrm{ms}$

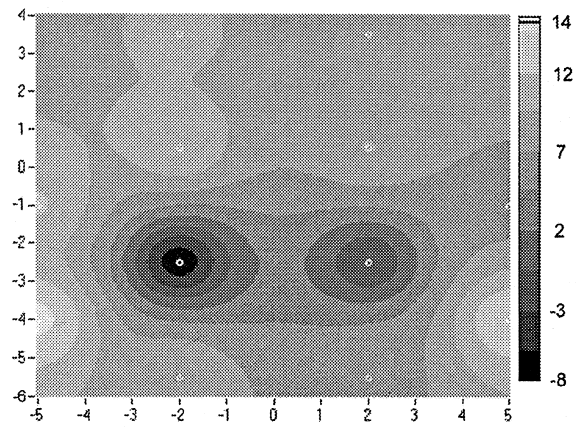

2. $\mathrm{T}=20.5 \mathrm{~ms}$
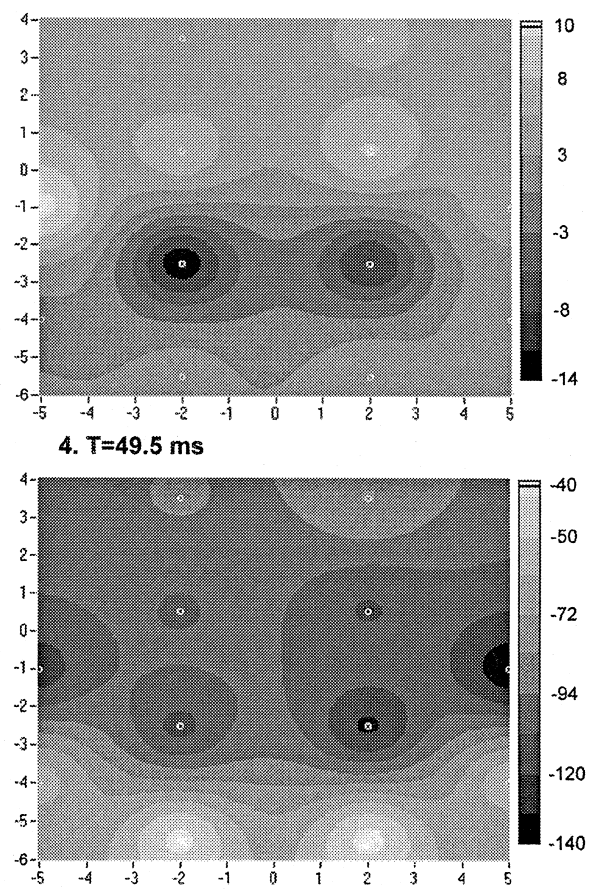
recorded using microwires $(n=3)$. Activities recorded from 1 of the implanted microwire bundles in response to laser irradiation of the tail-base are illustrated in Fig. 9. Responses of individual trials are shown at the top portion of Fig. 9A. Short and long latency intracortical responses could be seen clearly in both the IMUA (Fig. 9B) and the average spike count (Fig. 9C). The temporal relationship of these short and long latency intracortical responses with the short and long latency cortical LEPs can be better appreciated by putting the LEP of another rat along with the IMUA traces (Fig. 10B, top trace). There were higher multiunit activities in recording sites located at the surface and $400-\mu \mathrm{m}$ and $1000-\mu \mathrm{m}$ deeps, 30-60 ms after laser irradiation. For the late component, there were heightened activities throughout the layers. In comparison, the intracortical response to mechanical stimulation of the same region of the tail was large throughout the cortex at 15-25 $\mathrm{ms}$, then it shifted to more superficial loci from 30 to 50 ms (Fig. 10A).

\section{Discussion}

In the present study, cortical responses to nociceptive inputs from the tail are described for the first time in conscious and freely moving rats. A short latency response corresponding to $\mathrm{A} \delta$ fibers and a long latency response corresponding to $\mathrm{C}$ fibers were observed. Interesting similarities and differences were found between these nociceptive cortical responses and the tactile cortical responses.

\subsection{Cortical response to innocuous stimulation}

In the present investigation, mechanical stimulation evoked early surface positive potentials in the tail representative area of the SI. These findings are consistent with earlier investigations $[1,21,33]$. This surface-positive component is associated with unit activity and current sinks in the middle cortical layers (layers III/IV), and appears to signal the initial thalamocortical activation of SI (Refs. [1,21,33]; Fig. 10A). In a wakeful condition, a larger and more widespread negative peak followed. It is interesting to note that this negative peak is very sensitive to anesthesia (dotted line in Fig. 1). Using topographical mapping analysis, we showed that a bilateral negative current sink was generated first at the tail area of SI (Fig. 6B), which then spread to frontoparietal areas. This negative peak appeared to be associated with current sinks in superficial cortical layers (see Fig. 10A). Our current findings support those of a previous study, which demonstrated that behaviorally relevant negative potentials may be associated with the most superficial cortical layers I/II in awake monkeys $[11,12]$. The somatosensory-evoked potential recorded in awake humans is similar to that observed in the awake monkey [10]. In general, these results indicate that an early positive peak occurs at SI, followed by a superficial current sink in and around the SI area.

The low-intensity electrical stimulation-evoked cortical potential has almost identical temporal and spatial patterns compared to those of MEP. Our data are consistent with results from humans, monkeys and rats [1,21,27,33]. A localized current sink was generated at the middle layer of the primary somatosensory cortex for the fast, near-field initial positive-evoked potential. A large negative-evoked potential followed in the conscious human or animal subjects. This negative component $[1,10-12]$ also has a localized origin in the primary somatosensory cortex but originates from more superficial layers.

\subsection{Cortical response to noxious stimulation}

In the present study, we found that $\mathrm{CO}_{2}$-laser stimulation evokes two major negative components in awake, freely moving rats. Using the same type of stimuli to stimulate paws of halothane-anesthetized rats, Kalliomaki et al. found a positive component with a conduction velocity at $0.7-0.8 \mathrm{~m} / \mathrm{s}$, i.e., a $\mathrm{C}$ fiber response. It is interesting to note that similar conduction velocity value $(0.7 \mathrm{~m} / \mathrm{s}$, Fig. 3) was observed in the present study. This late negative component reversed to a positive wave when the rat was under light anesthesia (Fig. 1, right panel). It is important to note that during this period (2-h after a standard i.p. pentobarbital dose), although a reversed form of the longer latency LEP had returned, there was no trace of the shorter latency LEP yet. The shorter latency component conducting at the $A \delta$ range seems to be even more sensitive to modification by anesthesia. Maybe this is why this component was not consistently observed in Kalliomaki et al. [21].

Our topographic mapping analysis also confirms that the $\mathrm{C}$ fiber-mediated LEP component occupies a more widespread area than those evoked by mechanical or electrical stimulation $[21,27]$. The current sink of the C fibermediated LEP component seems to involve not only the primary somatosensory region but also the motor region of the cerebral cortex (Ref. [21]; Fig. 8). Interestingly, the

Fig. 7. Spatial distribution of EEPs. (A) A representative example of 12-channel recording of EEP stimulation intensity ( $0.5 \mathrm{~mA})$ is displayed in related recording sites over the skull (indicated at the inset). Each trace represents the average of 100 trials. The units of the $x$-axis and $y$-axis are 50 ms and 100 $\mu \mathrm{V}$, respectively. (B) Topographical maps of the EEPs. The upper trace is the EEP at channel 5 (tail representative area of SI). Four isopotential maps were made at latencies of 16,20.5, 27, and $49.5 \mathrm{~ms}$ from the start of the electrical stimulation, respectively. Note the localized peaks at latencies of $16,20.5$, and $27 \mathrm{~ms}$. The longer latency peak at $49.5 \mathrm{~ms}$ is represented in a widely distributed area. The unit of the gray scale is in microvolts $(\mu \mathrm{V})$. The units of the horizontal and vertical scales are in millimeters $(\mathrm{mm})$. The zero point is at the bregma. 

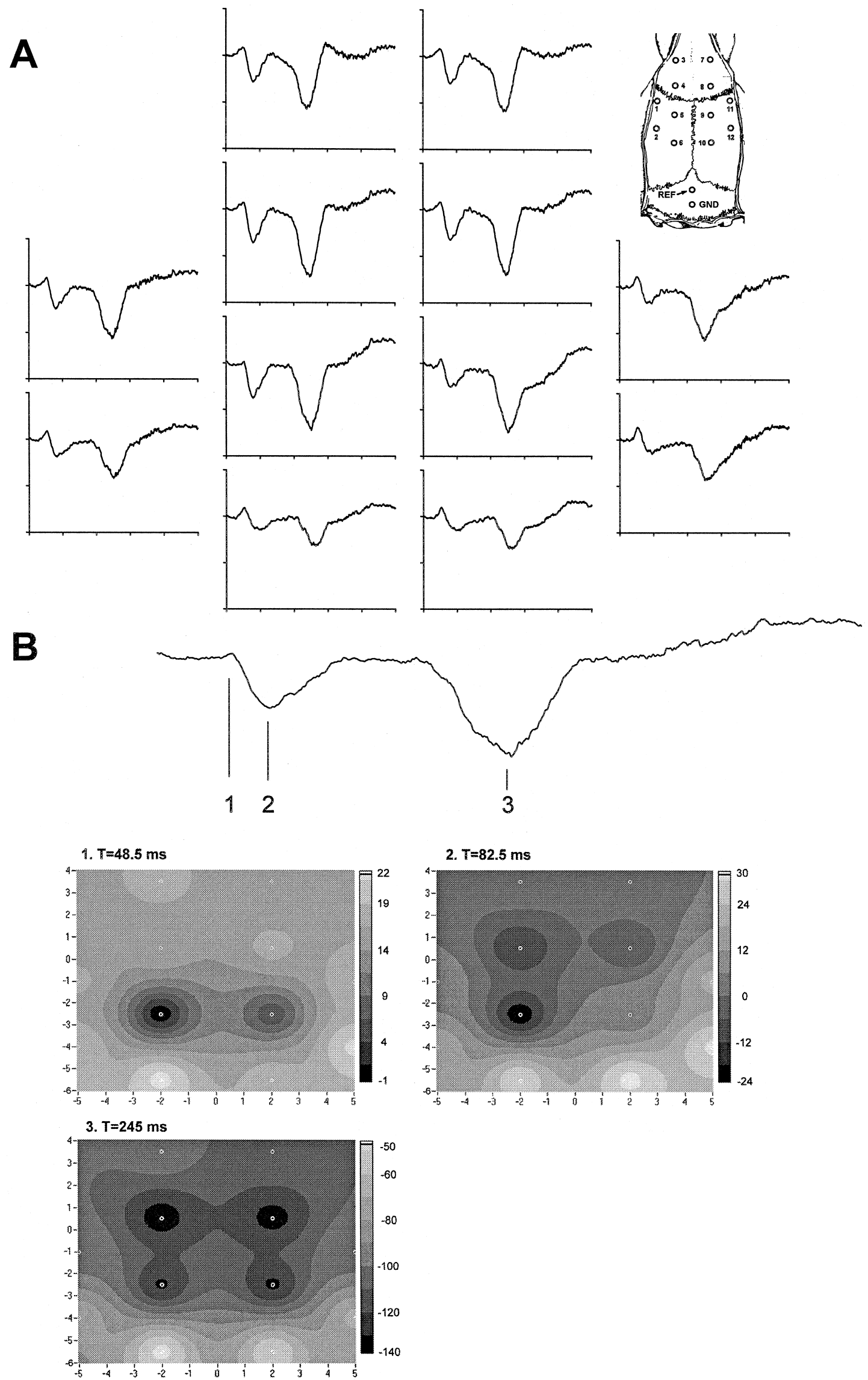
Table 1

Summary of spatiotemporal characteristics of MEP, EEP and LEP in freely moving rats

\begin{tabular}{|c|c|c|c|c|c|}
\hline & Component & $\begin{array}{l}\text { Polarity of } \\
\text { major peak }\end{array}$ & $\begin{array}{l}\text { Latency from } \\
\mathrm{Tb}(\mathrm{ms})\end{array}$ & $\begin{array}{l}\text { Conduction } \\
\text { velocity }(\mathrm{m} / \mathrm{s})\end{array}$ & Spatial distribution \\
\hline \multirow{2}{*}{ MEP } & 1 & Positive & $15.6 \pm 0.6$ & 35 & Concentric at SI \\
\hline & 2 & Negative & $49.5 \pm 2.0$ & 28 & Concentric at SI \\
\hline & 2 & Negative & $52.2 \pm 1.2$ & NA & Concentric at SI \\
\hline \multirow[t]{2}{*}{ EEP (high intensity) } & 1 & Positive & $16.9 \pm 0.5$ & NA & Concentric at SI \\
\hline & 2 & Negative & $50.3 \pm 0.8$ & NA & Diffuse \\
\hline
\end{tabular}

(1) Low intensity: $0.3 \mathrm{~mA}$; high intensity: $0.5 \mathrm{~mA}$.

(2) Values are mean \pm S.E.

(3) MEP: mechanical-evoked potential; EEP: electrical-evoked potential; LEP: laser-evoked potential; Tb: tail base; NA: not available.

same topographical distribution was found for the $\mathrm{A} \delta$ fiber-mediated LEP component (Fig. 8). These spatial patterns indicate a close association of sensorimotor integration for noxious information processing.

The intracortical data obtained in the present study show that cortical neurons are activated during both LEP components. This is supportive evidence that these components are cortical in origin. These data also add to the intracortical recording literature [21,28,29]. Our data are consistent with the laminar distribution of a middle and a deeper layer of cortical neuron activation from $\mathrm{C}$ fiber inputs in anesthetized rats [21]. In addition, we found heightened activities in the superficial cortical layer following $\mathrm{C}$ fiber activation in freely moving rats. Superficial, middle, and deeper layers of cortical neurons were also activated by $\mathrm{A} \delta$ fiber input (Fig. 10B), with the difference that cortical activation evoked from $\mathrm{C}$ fiber input lasted for a longer period. The result is also consistent with previous investigations by Lamour et al. [28,29], which demonstrated a few superficial neurons responsive to nociceptive stimulation. Percentage-wise, nociceptive superficial cortical neurons were relatively rare in halothane-anesthetized rats $[28,29]$. Our data indicate that there may be many more superficial cortical neurons responsive to nociceptive input in awake and freely moving rats.

\subsection{Comparison of cortical innocuous and noxious re- sponses}

Similarities and differences were found in cortical responses to innocuous mechanical and noxious thermal stimulation. For both stimuli, the most pronounced responses were seen in the matched area of the primary somatosensory cortex. These larger responses over the primary somatosensory cortex showed intensity-dependent changes in magnitude, indicating their possible utility in the sensory-discrimination function of tactile and pain senses. On the other hand, the slower negative cortical wave seen after strong electrical current or noxious thermal heat application to the tail had a more widespread distribution over the cortical surface than did tactile-evoked potentials. The present study also reveals interesting differential modulation actions by barbiturate anesthesia. The first positive peak evoked by innocuous mechanical stimulation was potentiated 5 times after a standard i.p. dose (50 $\mathrm{mg} / \mathrm{kg}$ ) of sodium pentobarbital. In sharp contrast, the faster $A \delta$ peak evoked by radiant heat disappeared completely for more than $3 \mathrm{~h}$ with the same barbiturate treatment [35]. Two hours after i.p. barbiturate administration, the slower component of the LEP returned but with a reversed polarity (dotted line in Fig. 1). Barbiturates act directly on vertebrate neurons by enhancing the effects of the inhibitory transmitter, $\gamma$-aminobutyric acid (GABA) $[2,40]$. Shyu et al. [36] found that pentobarbital abolishes field potentials of the superficial cortical layer without affecting those of the middle cortical layer for central lateral nucleus stimulation in chloralose-anesthetized cat. Other studies $[10,38]$ also indicate that activities of neurons in the superficial layer of the cerebral cortex are very sensitive to anesthesia. Selectively abolishing superficial layer activity more than the activity in deeper cortical layers may cause the polarity changes observed in the $\mathrm{A} \delta$ and $\mathrm{C}$ fiber-evoked potentials during pentobarbital anesthe-

Fig. 8. Spatial distribution of LEPs. (A) An example of 12-channel recordings of LEPs (12 W at tail base) displayed in related recording sites over the skull (indicated at the inset). Each trace represents the average of more than 50 trials. The units of the $x$-axis and $y$-axis are $100 \mathrm{~ms}$ and $100 \mu \mathrm{V}$, respectively. (B) Topographical map of the LEPs. The upper trace is the LEP from channel 5. Three isopotential maps are made at latencies of 48.5, 82.5, 245 ms from the start of the $\mathrm{CO}_{2}$-laser stimulation point, respectively. Note a localized distribution $(T=48.5 \mathrm{~ms})$ followed by a widespread distributed component $(T=82.5 \mathrm{~ms})$. A widespread potential distribution was also found for the second negative component $(T=245 \mathrm{~ms})$. The unit of the gray scale is in microvolts $(\mu \mathrm{V})$. The units of the horizontal and vertical scales are in millimeters $(\mathrm{mm})$. The zero point is at the bregma. 
A

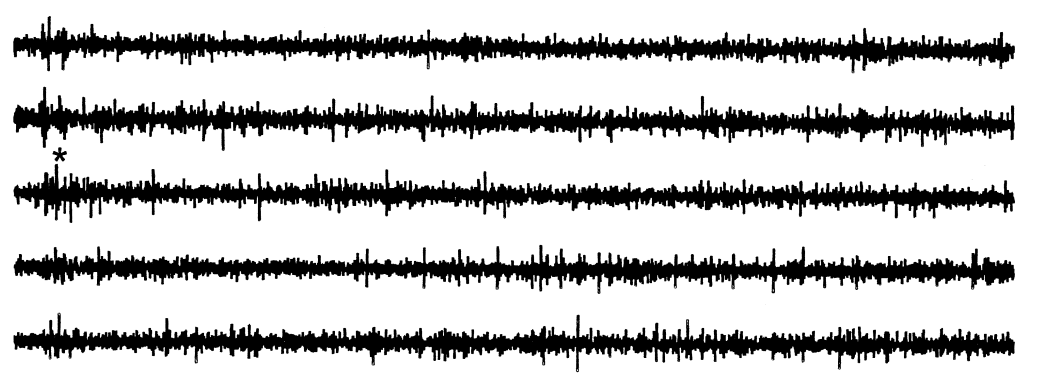

*
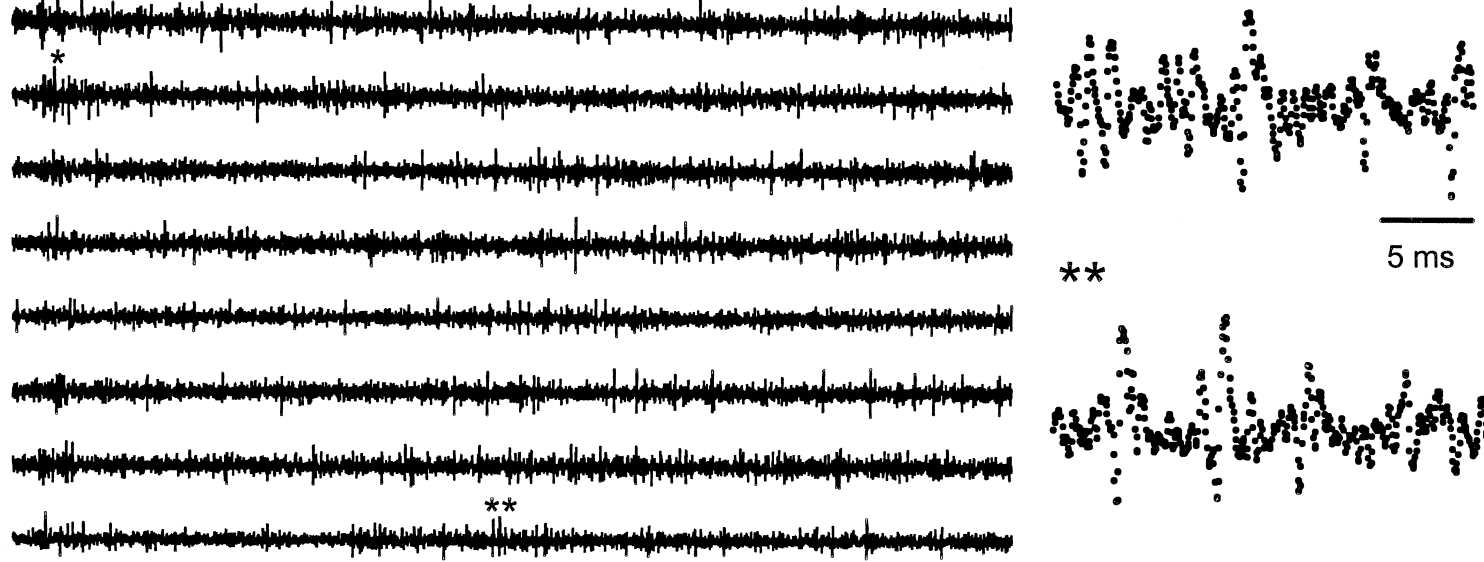

*
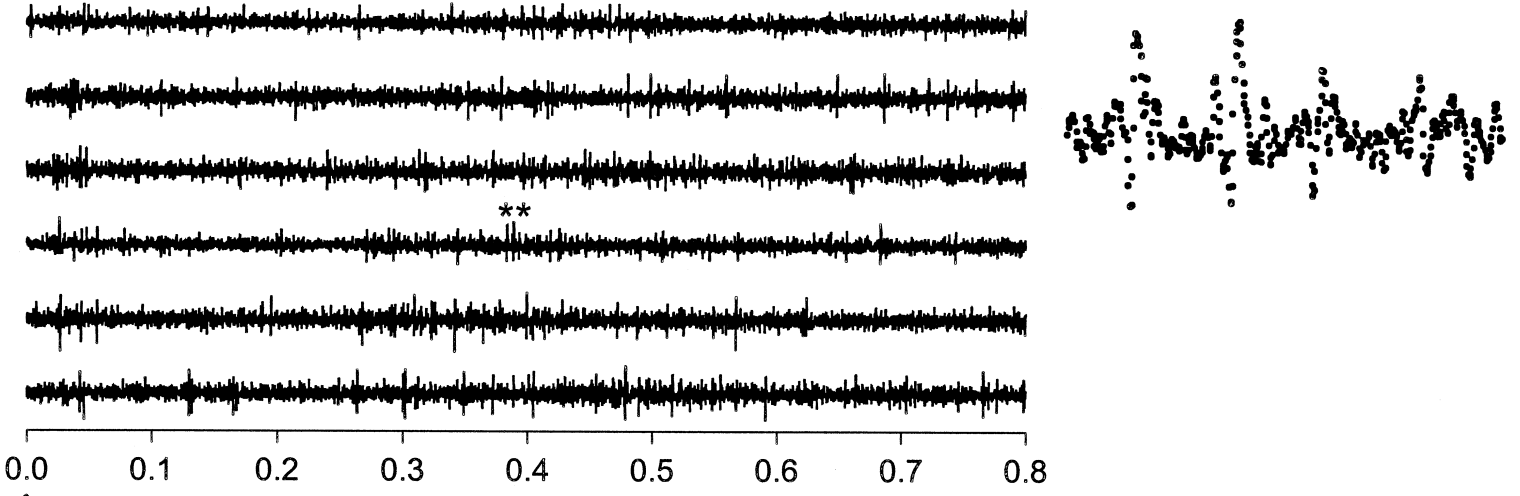

B $\uparrow$ Time (s)
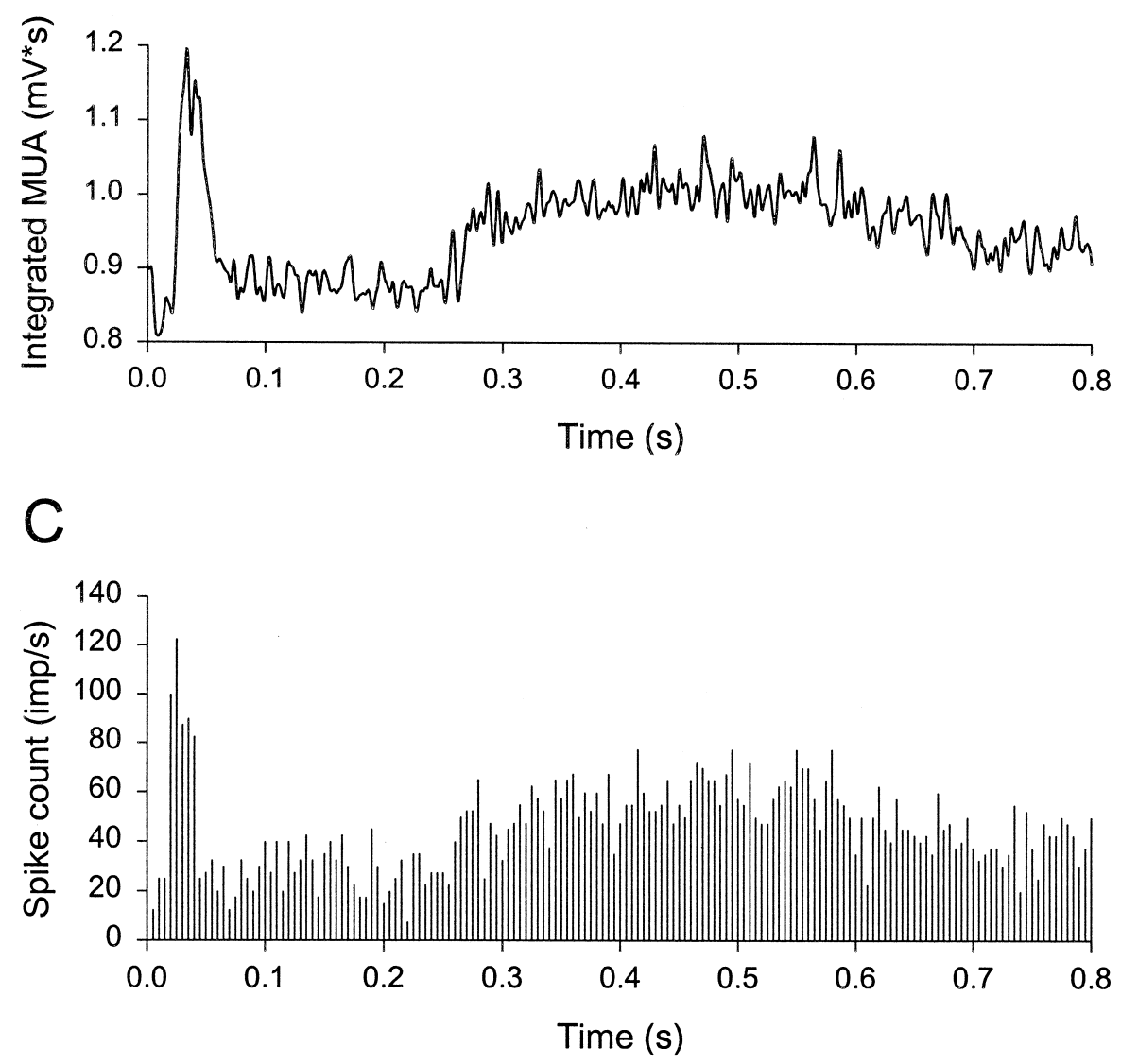

Fig. 9. Response of intracortical multiunit activity to laser irradiation (15 W) of the middle tail region. (A) Eleven individual trials. Arrow points to the start of the stimulation. On the right are expanded views of two segments relative to the two components of LEP ( $*$ first component; $* *$ second component). Note multiunit (sampling rate: $30 \mathrm{kHz}$ ) activities. (B) Averaged (90 trials) integrated rectified multiunit activity (MUA) obtained from the same experiment. (C) Multiunit spike counts obtained from the same set of data. 


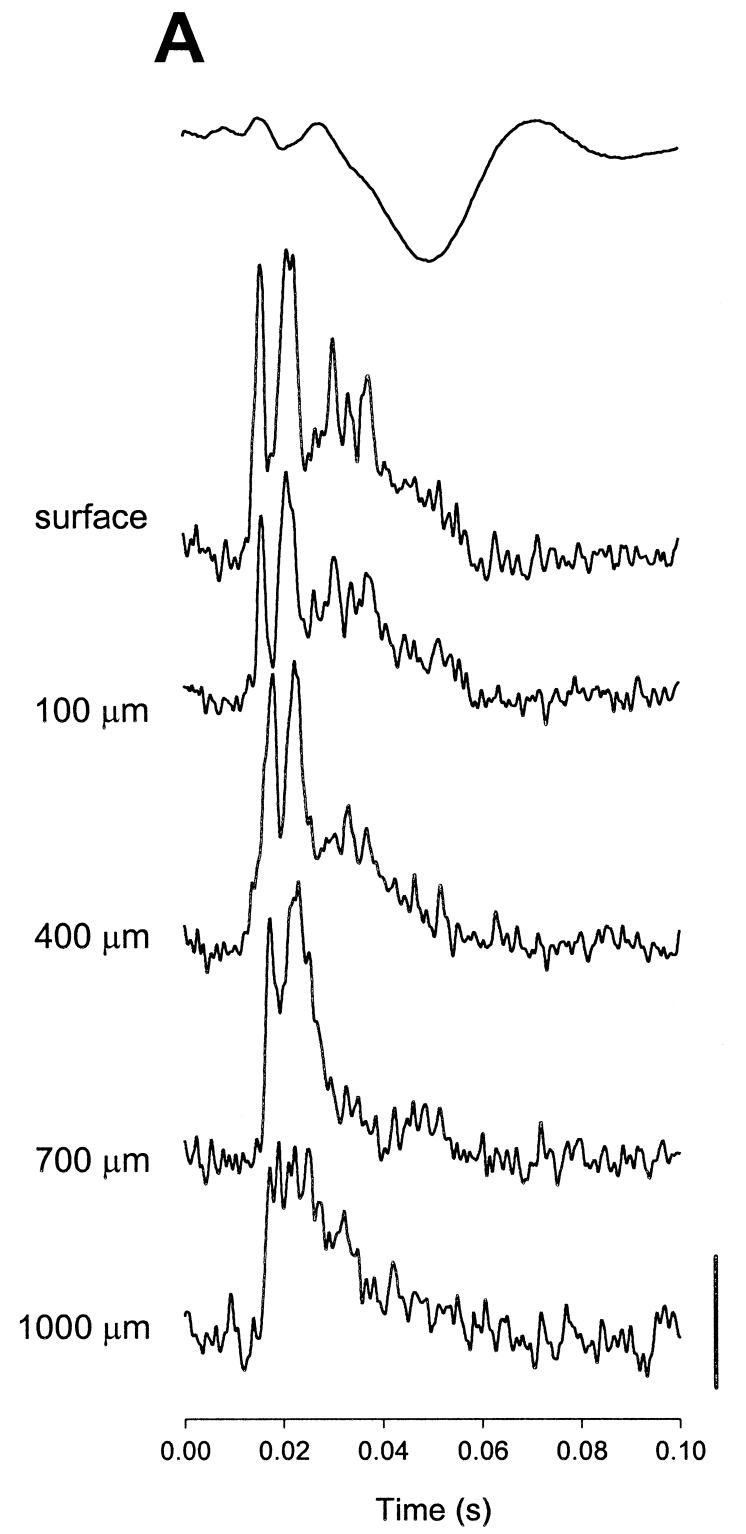

B
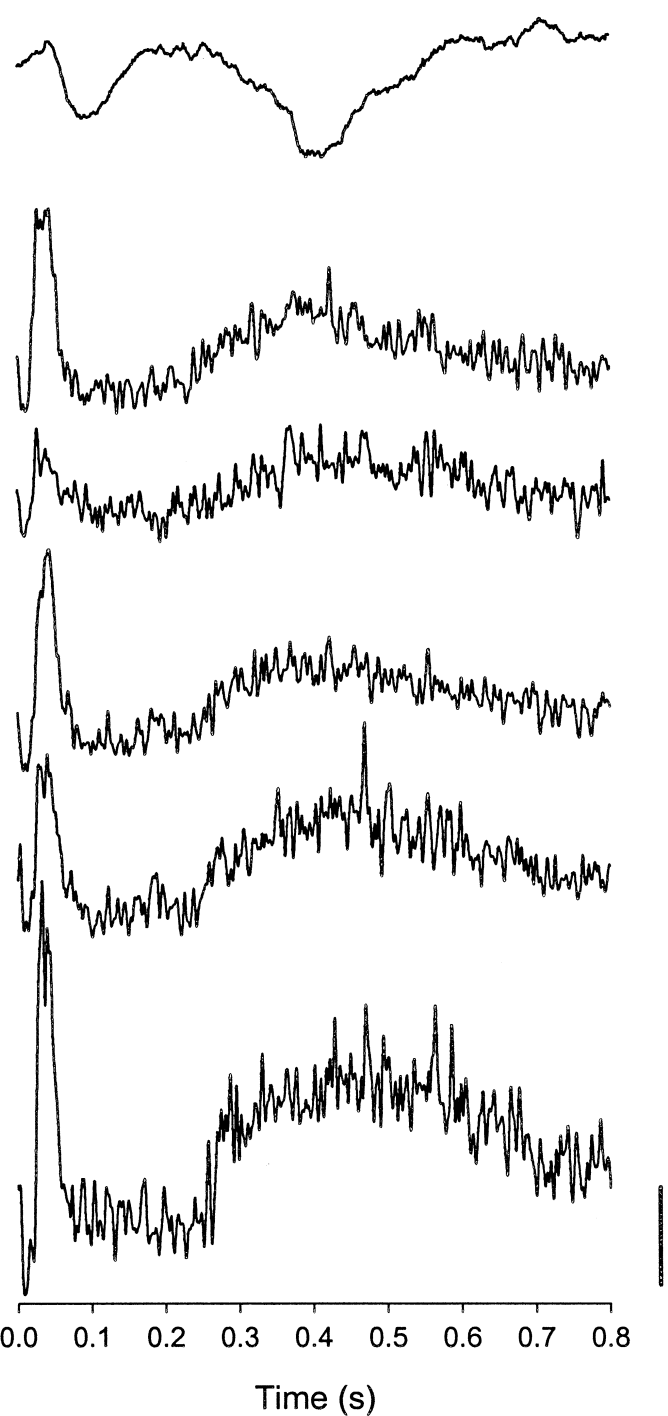

Fig. 10. Responses of intracortical multiunit recording at different depths following mechanical (A) and laser heat (B) stimulation at the middle tail. Recording depth is indicated on the left side. Each trace represents averaged rectified and integrated multiunit activity from more than 50 trials. The vertical bars are $0.5 \mathrm{mV}$ s for (A), $0.1 \mathrm{mV}$ s for (B), and $10 \mu \mathrm{V}$ for (C).

sia in the present study. The other possibility is that barbiturate modulates the activity of the nociceptive input or the ascending nociceptive pathway [13,26,38].

In summary, with chronically implanted cortical electrodes, two negative cortical potentials were found following laser stimulation of tails of awake and freely moving rats. Peripheral conduction velocity calculations indicate that these two evoked potentials correspond to $\mathrm{A} \delta$ and $\mathrm{C}$ fiber activation, respectively. These negative potentials, in contrast to the robust short latency positive potential, are highly susceptive to modulation by barbiturate anesthesia. Because of this state dependency, these cerebral potentials might be related to the cognitive and affective part of the pain sensation.

\section{Acknowledgements}

We thank Professors R.C.S. Lin and J.K. Chapin, Allegheny University, USA, for their comments. We thank Mr. Daniel P. Chamberlin for English editing. The present study was supported by grants NSC84-2311-B002-027 and NSC86-2313-B002-123 from the National Science Council of the Republic of China (Taiwan).

\section{References}

[1] J.C. Arezzo, H.G. Vaughan Jr., A.D. Legatt, Topography and intracranial sources of somatosensory evoked potentials in the mon- 
key: II. Cortical components, Electroencephalogr. Clin. Neurophysiol. 28 (1981) 1-18.

[2] J.L. Baker, D.A. Mathers, GABA analogues activate channels of different duration on clustered mouse spinal neurons, Science 212 (1981) 358-361.

[3] B. Bromm, H. Neitzel, A. Tecklenburg, R.-D. Treede, Evoked cerebral potential correlates of $\mathrm{C}$ fiber activity in man, Neurosci. Lett. 43 (1983) 109-114.

[4] B. Bromm, J.E. Desmedt (Eds.), Pain and the brain, from nociception to cognition, Advances in Pain Research and Therapy, Vol. 22, Raven, New York, 1995.

[5] B. Bromm, M.T. Jahnke, R.-D. Treede, Responses of human cutaneous afferents to $\mathrm{CO}_{2}$-laser stimuli causing pain, Exp. Brain Res. 55 (1984) 158-166.

[7] B. Bromm, R.-D. Treede, Human cerebral potentials evoked by $\mathrm{CO}_{2}$-laser stimuli causing pain, Exp. Brain Res. 67 (1987) 153-162.

[8] J.N. Campbell, S.N. Raja, R.A. Meyer, Halothane sensitizes cutaneous nociceptors in monkeys, J. Neurophysiol. 52 (1984) 762-770.

[9] A. Carmon, Y. Dotan, Y. Sarne, Correlation of subjective pain experience with cerebral evoked responses to noxious thermal stimulation, Exp. Brain Res. 33 (1978) 445-453.

[10] L.J. Cauller, Layer I of primary sensory neocortex: where top-down converges upon bottom-up, Behav. Brain Res. 71 (1995) 163-170.

[11] L.J. Cauller, A.T. Kulics, A comparison of awake and sleeping cortical states by analysis of the somatosensory-evoked response of postcentral area 1 in rhesus monkey, Exp. Brain Res. 72 (1988) 584-592.

[12] L.J. Cauller, A.T. Kulics, The neural basis of the behaviorally relevant N1 component of the somatosensory-evoked potential in SI cortex of awake monkeys: evidence that backward cortical projections signal conscious touch sensation, Exp. Brain Res. 84 (1991) 607-619.

[13] J.K. Chapin, B.D. Waterhouse, D.J. Woodward, Differences in cutaneous sensory response properties of single somatosensory cortical neurons in awake and halothane anesthetized rats, Brain Res. Bull. 6 (1981) 63-70.

[14] J.K. Chapin, C.-S. Lin, The somatic sensory cortex of the rat, in: B. Kolb, R.C. Tees (Eds.), The Cerebral Cortex of the Rat, MIT, Cambridge, 1990, pp. 341-380.

[15] M. Devor, A. Carmon, R. Frostig, Primary afferent and spinal sensory neurons that respond to brief pulses of intense infrared laser radiation: a preliminary survey in rats, Exp. Neurol. 76 (1982) 483-494.

[16] W.K. Dong, L.D. Salonen, Y. Kawakami, T. Shiwaku, M. Kaukoranta, R.F. Martin, Nociceptive responses of trigeminal neurons in SII-7b cortex of awake monkey, Brain Res. 484 (1989) 314-324.

[17] H. Head, G. Holms, Sensory disturbances from cerebral lesions, Brain 34 (1911) 102-254.

[18] R.F. Hellon, N.K. Misra, K.A. Provins, Neurons in the somatosensory cortex of the rat responding to scrotal skin temperature changes, J. Physiol. 232 (1973) 401-411.

[20] A.K. Jones, W.D. Brown, K.J. Friston, L.Y. Qi, R.S. Frackowiak, Cortical and subcortical localization of response to pain in man using positron emission tomography, Proc. R. Soc. London B 244 (1991) 39-44.

[21] J. Kalliomaki, H.-R. Weng, H.-J. Nilsson, J. Schouenborg, Nociceptive $\mathrm{C}$ fiber input to the primary somatosensory cortex (SI). A field potential study in the rat, Brain Res. 622 (1993) 262-270.

[22] D.R. Kenshalo, E.H. Chudler, F. Anton, R. Dubner, SI nociceptive neurons participate in the encoding process by which monkey perceive the intensity of noxious thermal stimulation, Brain Res. 454 (1988) 378-382.

[23] D.R. Kenshalo, O. Isensee, Responses of primate SI cortical neurons to noxious stimuli, J. Neurophysiol. 50 (1983) 1479-1496.

[24] D.R. Kenshalo, W.D. Willis, The role of cerebral cortex in pain system, in: A. Peter, E.G. Jones (Eds.), Cerebral Cortex: Normal and Altered States of Function, Vol. 9, Plenum, New York, 1991, pp. $153-212$.

[25] Y. Kitamura, R. Kakigi, M. Hoshiyama, S. Koyama, M. Shimojo, S. Watanabe, Pain-related somatosensory evoked magnetic fields, Electroencephalogr. Clin. Neurophysiol. 95 (1995) 463-474.

[26] E. Kochs, R.-D. Treede, J. Schulte am Esch, B. Bromm, Modulation of pain-related somatosensory evoked potentials by general anesthesia, Anesth. Analg. 71 (1990) 225-230.

[27] V. Kunde, R.-D. Treede, Topography of middle-latency somatosensory evoked potentials following painful laser stimuli and non-painful electrical stimuli, Electroencephalogr. Clin. Neurophysiol. 88 (1993) 280-289.

[28] Y. Lamour, G. Guilbaud, J.C. Willer, Rat somatosensory (SmI) cortex: II. Laminar and columnar organization of noxious and non-noxious inputs, Exp. Brain Res. 49 (1983) 46-54.

[29] Y. Lamour, J.C. Willer, G. Guilbaud, Rat somatosensory (SmI) cortex: I. Characteristics of neuronal responses to noxious stimulation and comparison with responses to non-noxious stimulation, Exp. Brain Res. 49 (1983) 35-45.

[30] N. Matsumoto, T. Sato, T.A. Suzuki, Characteristics of the tooth pulp driven neurons in a functional column of the cat somatosensory cortex (SI), Exp. Brain Res. 74 (1988) 263-271.

[31] F. Perrin, J. Pernier, O. Bertrand, M.H. Giard, J.F. Echallier, Mapping of scalp potentials by surface spline interpolation, Electroencephalogr. Clin. Neurophysiol. 66 (1987) 75-81.

[32] A. Roos, B. Rydenhag, S.A. Andersson, Cortical responses evoked by tooth pulp stimulation in the cat. Surface and intracortical responses, Pain (1982) 247-265.

[33] J. Schouenborg, J. Kalliomaki, P. Gustavsson, I. Rosen, Field potentials evoked in the rat somatosensory cortex (SI) by impulses in cutaneous A $\beta$ - and C-fibres, Brain Res. 397 (1986) 86-92.

[34] F.-Z. Shaw, R.-F. Chen, E.H.-Y. Lee, H.-W. Tsao, C.-T. Yen, Mapping of cortical field potentials evoked by electrical, mechanical and laser heat stimulation of the rat tail, Soc. Neurosci. Abstr. 23 (1997) 440.

[35] F.-Z. Shaw, R.-F. Chen, H.-W. Tsao, C.-T. Yen, A multichannel system for recording and analysis of cortical field potentials in freely moving rats, J. Neurosci. Methods (in press).

[36] B.C. Shyu, B. Olausson, B. Rydenhag, Field potential analysis of the cortical projection of the central lateral nucleus in the cat, Acta Physiol. Scand. 137 (1989) 503-512.

[37] J.D. Talbot, S. Marrett, A.C. Evans, E. Meyer, M.C. Bushnell, G.H. Duncan, Multiple representations of pain in human cerebral cortex, Science 251 (1991) 1355-1358.

[38] B.A. Vogt, The role of layer I in cortical function, in: A. Peter, E.G. Jones (Eds.), Cerebral Cortex: Normal and Altered States of Function, Vol. 9, Plenum, New York, 1991, pp. 49-80.

[39] C.-T. Yen, C.-H. Huang, S.-E. Fu, Surface temperature change, cortical evoked potential and pain behavior elicited by $\mathrm{CO}_{2}$ lasers, Chin. J. Physiol. 37 (1994) 193-199.

[40] S.A. Zimmerman, M.V. Jones, N.L. Harrison, Potentiation of $\gamma$ aminobutyric $\operatorname{acid}_{\mathrm{A}}$ receptor $\mathrm{Cl}^{-}$current correlates with in vivo anesthetic potency, J. Pharmacol. Exp. Ther. 270 (1994) 987-991. 